\title{
Oscillations in Intracellular ATP, cAMP and cGMP Concentration in Relation to Rhythmical Sporulation under Continuous Light in the Myxomycete Physarum polycephalum
}

\author{
By T. AKITAYA, S. OHSAKA, T. UEDA* AND Y. KOBATAKE \\ Faculty of Pharmaceutical Sciences, Hokkaido University, Sapporo 060, Japan
}

(Received 4 July 1984)

\begin{abstract}
The times of sporulation in populations of plasmodia derived from single starving plasmodia and the variation in intracellular ATP, cAMP and cGMP concentrations were determined from the time when the plasmodia were exposed to continuous light. Sporulation occurred from about $10 \mathrm{~h}$ after illumination, with further intermittent sporulation at $5 \mathrm{~h}$ or $10 \mathrm{~h}$ intervals. Intracellular ATP, cAMP and cGMP concentrations oscillated, usually in phase, with a period of 4-5 h until the irreversible commitment to sporulation occurred. The ATP concentration stopped oscillating, remained at the same level for a few hours and decreased gradually during sporangium formation. Oscillations in cAMP and cGMP concentrations continued, little affected by commitment to sporulate or subsequent sporulation. The period of ATP oscillation did not differ over a wide range of temperature. In plasmodia which were starved only for $1 \mathrm{~d}$ and therefore unable to sporulate, light failed to induce ATP oscillation. The concentrations of cAMP and cGMP oscillated but not in phase.
\end{abstract}

\section{INTRODUCTION}

Sporulation in Physarum polycephalum is a differentiation process which a starving plasmodium undergoes after illumination. Because the changes associated with sporulation occur synchronously throughout a large plasmodium, this system is useful for studying events during differentiation both biochemically and biophysically (Sauer et al., 1969; Sauer, 1982; Goodman, 1980; Gorman \& Wilkins, 1980). In many sporulation experiments, plasmodia are cultured axenically in a semi-defined medium, transferred to a non-nutrient medium containing niacin, and kept in a dark place for several days (Daniel \& Rusch, 1962a,b). Then, after a $4 \mathrm{~h}$ illumination period the plasmodium is irreversibly committed to sporulate: nodules appear at intervals on the veins and these quickly form pillars which thereafter develop sporangia at their apex. The final stages of sporulation involve deposition of melanin pigment, cleavage of apical cytoplasm, meiosis and spore formation (Guttes et al., 1961; Dee, 1975).

Here we examine the sporulation process in oat-cultured plasmodia (Hosoda, 1980) by measuring intracellular concentration of ATP, cAMP and cGMP in various experimental conditions. Concentrations of all these components oscillate, the oscillation being related to an intermittent formation of sporangia under continuous light.

\section{METHODS}

Organism. A plasmodial strain from Carolina Biological Supply was sporulated and spores germinated to produce amoebae. After establishing new cloned amoeba strains, a diploid plasmodial strain (HU $195 \times$ HU 200) was produced by mating amoebae. A second strain used was a haploid albino plasmodial strain (LU 404). Plasmodia were fed on oatflakes and stored as sclerotia.

Starvation conditions. Plasmodia were transferred either to water agar $(1 \%)$ or to filter paper on water agar in a trough $(30 \mathrm{~cm} \times 40 \mathrm{~cm})$ without food and kept in darkness at $21 \pm 1{ }^{\circ} \mathrm{C}$. The plasmodium wet weight was about 
$7 \mathrm{~g}$, its advancing front extending the full width of the trough. Plasmodia were adapted to experimental temperatures for $1 \mathrm{~h}$ prior to illumination.

Induction of sporulation under continuous light. After a period of starvation, plasmodia were illuminated by fluorescent tubes $(30 \mathrm{~W} \times 2)$ from $30 \mathrm{~cm}$ above. The light intensity was about $5.3 \mathrm{~W} \mathrm{~m}^{-2}$ (Radiometer, Model 65) which gradually bleached the yellow pigments of the plasmodium.

Determination of sporulation frequency as a function of time. A large starving plasmodium migrating on filter paper was cut into 30-50 pieces (approx. $1 \mathrm{~cm}^{2}$ ) together with underlying filter paper, and each piece transferred onto filter papers (five sheets thick, Whatman no. 1) moistened by distilled water in a $9 \mathrm{~cm}$ Petri dish. Filter paper was used because it is non-nutrient; agar can be utilized as a carbon source by $P$. polycephalum (Knowles \& Carlile, 1978). The plasmodium formed a continuous network of plasmodial veins. The first visible morphological change was the development of papillae which rose above the substratum and subsequently became separated from the network. This transition from continuous to isolated morphology was complete within $30 \mathrm{~min}$. This rapid process was utilized as the time of onset of sporulation. The number of dishes in which the process took place was noticed at $1 \mathrm{~h}$ intervals up to $50 \mathrm{~h}$ after illumination in order to determine the timing of sporulation.

Determination of intracellular ATP, cAMP and cGMP concentrations following illumination. A small frontal part (about $5 \mathrm{~mm}^{2}$ ) of a large plasmodium migrating on an agar gel was excised together with underlying agar. For the extraction of ATP, the plasmodial piece was immersed in $4 \mathrm{ml}$ of hot $10 \mathrm{mM}-\mathrm{Tris} / \mathrm{HCl}\left(\mathrm{pH} 7 \cdot 0,90-95^{\circ} \mathrm{C}\right)$ for 10 min. The suspension was centrifuged $(15000 \mathrm{~g}$ ) for $10 \mathrm{~min}$. The amount of ATP in the supernatant was determined photometrically by the luciferin-luciferase method with firefly lantern (Beutler \& Baluda, 1964). The pellet was used for the protein assay by the Lowry method. The ATP concentrations in plasmodia before illumination were $19 \cdot 5-33 \cdot 5 \mathrm{nmol}$ (mg protein $)^{-1}$.

For cAMP and cGMP measurements, the plasmodial piece was immersed in isopentane cooled by liquid $\mathrm{N}_{2}$, transferred to $1 \mathrm{ml}$ ice-cooled $1 \mathrm{M}-\mathrm{HCl}$, homogenized and washed twice with $0.5 \mathrm{ml}$ ice-cooled $1 \mathrm{M}-\mathrm{HCl}$. The homogenate was centrifuged $(15000 \mathrm{~g})$ for $10 \mathrm{~min}$. The supernatant was used for cAMP and cGMP assay with the Yamasa Assay Kit based on the method of Honma et al. (1977) and obtained from Yamasa Shoyu K. K., Choshi 288, Japan. The pellet was used for the protein assay. The intracellular concentrations of cAMP and cGMP before illumination varied from culture to culture. For plasmodia starved for $4 \mathrm{~d}$ the range was from $4 \cdot 0-30 \mathrm{pmol}$ cAMP (mg protein $)^{-1}$ and $1.5-7.5$ pmol cGMP (mg protein $)^{-1}$. For simultaneous measurements of ATP, cAMP and cGMP the plasmodial piece was halved, one for ATP assay and the other for cyclic nucleotide assays. The time courses shown are typical, chosen from 3-4 results for cyclic nucleotides and from 5-16 results for ATP at each experimental condition.

Determination of correlation coefficient between two oscillating quantities. Suppose that two quantities $x$ and $y$ oscillate or fluctuate with time, and we want to know the phase relationship between the two. The correlation coefficient, $\operatorname{cor}(x, y)$, is a quantitative measure of this, and is defined as $(\langle x y\rangle-\langle x\rangle\langle y\rangle) /\left(\sigma_{x} \sigma_{y}\right)$ where \langle\rangle represents means, and $\sigma_{x}$ and $\sigma_{y}$ are the standard deviations of $x$ and $y$, respectively. $\operatorname{Cor}(x, y)$ takes values between -1 and 1 ; there is a strong correlation when $\operatorname{cor}(x, y)$ is close to 1 , because $\operatorname{cor}(x, x)=1$. There is no correlation (i.e. $x$ and $y$ are independent) when cor $=0$. Correlation between cAMP and cGMP oscillations was examined.

\section{RESULTS AND DISCUSSION}

\section{Intermittent sporulation under continuous light}

Plasmodia, following starvation for times ranging from $100 \mathrm{~h}$ to $173 \mathrm{~h}$, were put under continuous light and the time to sporulation noted (Fig. 1). Sporulation sometimes occurred $10 \mathrm{~h}$ after illumination. Sometimes all the plasmodia derived from a single large plasmodium Fig. $1(b, h)$ sporulated simultaneously. Sometimes several peaks of sporulation were observed, with about $5 \mathrm{~h}$ or $10 \mathrm{~h}$ intervals. Plasmodia starved for less than $3 \mathrm{~d}$ prior to illumination did not bear spores, in agreement with the observations of Chapman \& Coote (1982). The rhythm of sporulation suggests that an oscillatory chemical reaction may control the sporulation process.

\section{Oscillations in cAMP, cGMP and ATP concentrations accompanying sporulation under continuous light}

Variations in intracellular cAMP, cGMP and ATP concentrations are shown in Fig. 2, where a plasmodium previously starved for $4 \mathrm{~d}$ was subdivided and then illuminated from time zero. After illumination, cAMP, cGMP and ATP concentrations began to oscillate in phase until shortly before commitment to sporulation. At this point ATP concentration stopped oscillating, stayed at a constant level for a few hours, and diminished gradually, accompanying the visible formation of sporangia. The two cyclic nucleotides continued to oscillate throughout 

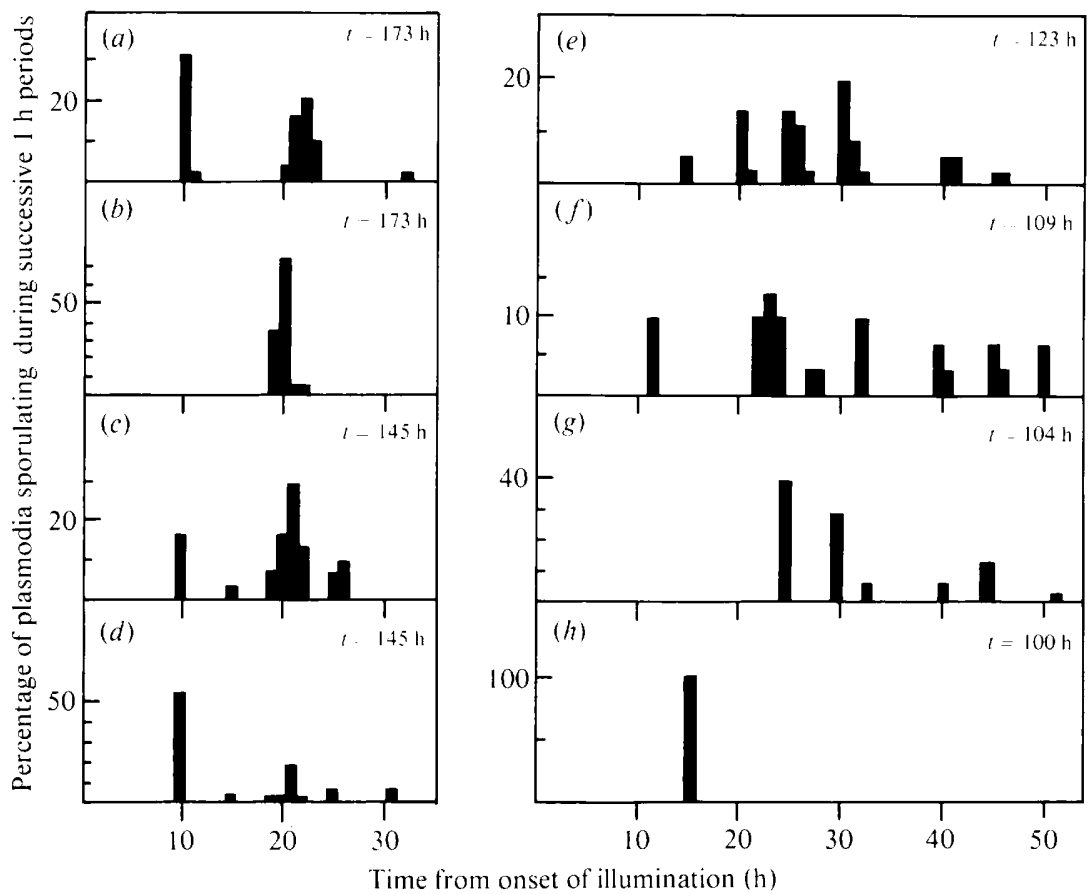

Fig. 1. Sporulation frequency as a function of time in Physarum plasmodia. Eight large plasmodia $(a-h)$ were each divided into $30-50$ pieces as follows: (a) 42, (b) 50, (c) 30, (d) 30, (e) 35, $(f) 30,(g) 30,(h) 50 . t$ is the duration of starvation prior to continuous illumination.

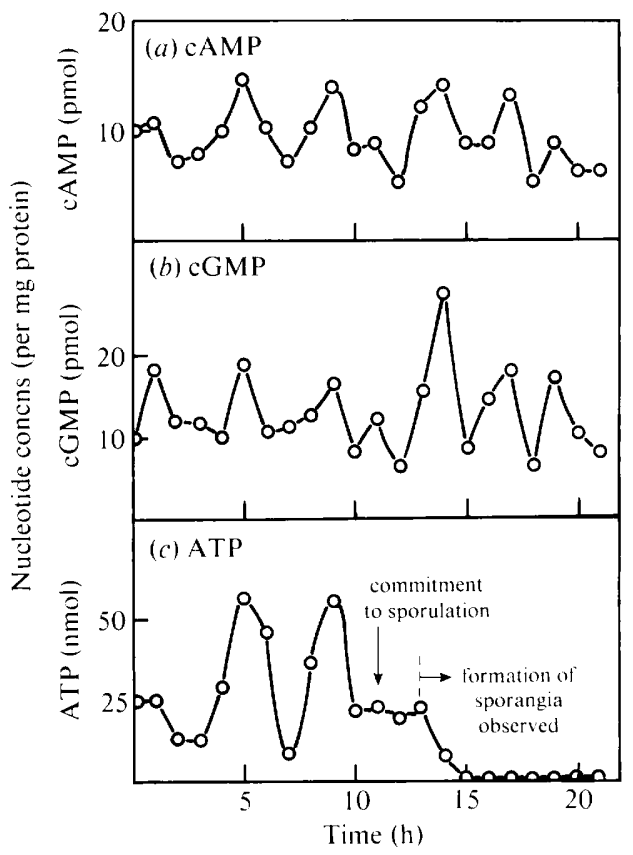

Fig. 2. Time courses of intracellular cAMP, cGMP and ATP concentrations, measured simultaneously, accompanying sporulation under continuous illumination. Commitment and sporulation occurred 11 and $13 \mathrm{~h}$ after illumination, respectively. 

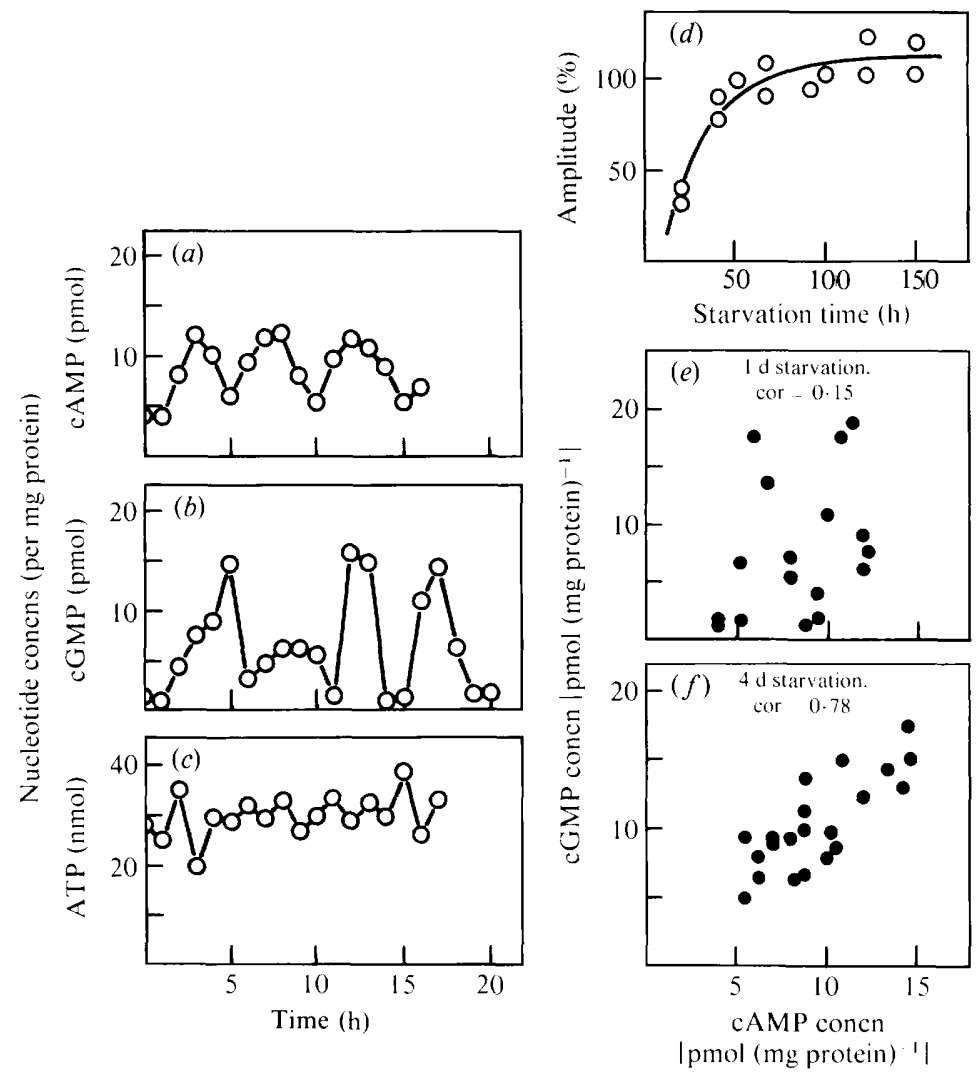

Fig. 3. Effects of starvation on light-induced cAMP, cGMP and ATP oscillations. $(a, b, c)$ Time courses of cAMP, cGMP and ATP oscillations following illumination of a plasmodium previously starved for $1 \mathrm{~d} ;(d)$ the relative amplitude, an average of peak-trough differences divided by the value at time zero of illumination for a plasmodium, of the ATP oscillation as a function of prior starvation time. Each data point was taken from different plasmodia; $(e, f)$ correlation between cAMP and cGMP oscillations in plasmodia starved for $1 \mathrm{~d}$ and $4 \mathrm{~d}$, respectively.

sporulation. Daniel (1966) had previously observed a fluctuation in ATP level in starving plasmodia, but his measurements were limited to a $5 \mathrm{~h}$ period immediately following the onset of illumination.

At the time of sampling for ATP assay, pieces of the plasmodia were cut off and put on wet oatflakes in complete darkness. Spore formation was observed. A commitment to sporulation was attained $11 \mathrm{~h}$ after illumination, corresponding closely to the cessation of ATP oscillation. This occurred a few hours earlier than the visible onset of sporulation: papilla formation. At this point ATP concentration began to diminish. In experiments in which commitment was attained later than that shown in Fig. 2(c) ATP oscillation continued to just before commitment. Thus, intracellular ATP concentration in the Physarum plasmodium is a sensitive indicator for events underlying morphogenetic processes, both visible and invisible.

\section{Effect of duration of prior starvation on light-induced oscillations of ATP, cAMP and CGMP concentrations}

The effect of the duration of prior starvation on variations in ATP, cAMP and cGMP concentration is shown in Fig. 3(a-c). With a short preceding starvation $(1 \mathrm{~d})$ there were only small and irregular fluctuations of ATP concentrations (Fig. $3 c$ ), while large and oscillatory variations of ATP were induced in plasmodia with prolonged preceding starvation, as shown in Fig. 2(c). The amplitude became larger as the preceding starvation period became longer, and 


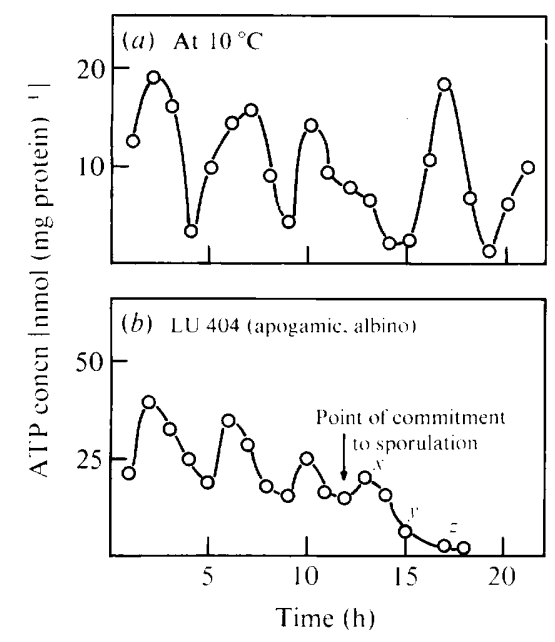

Fig. 4. Time courses of intracellular ATP concentrations following illumination. (a) Plasmodium of HU $195 \times$ HU 200 transferred to $10^{\circ} \mathrm{C} 1 \mathrm{~h}$ before illumination; $(b)$ plasmodium of the apogamic albino strain LU 404 at $22^{\circ} \mathrm{C}$. Papilla formation began at $x$, melanization took place at $y$, and sporangia were formed at $z$.

reached about twice the level of prior illumination with 3 day starvation (Fig. $3 d$ ). The period of the oscillation, once established, was from 4 to $5 \mathrm{~h}$, for all the starvation treatments employed. This acquisition through starvation of the ability to undergo ATP oscillations in response to light appears to correspond to that of the ability to sporulate in response to light.

Variations in cAMP and cGMP concentrations following $1 \mathrm{~d}$ starvation prior to illumination were also measured (Fig. $3 a, b$ ). The two cyclic nucleotides oscillated with a period of $4-5 \mathrm{~h}$ as with $4 \mathrm{~d}$ prior starvation. The amplitude of cGMP oscillation was somewhat larger than that in plasmodia starved for $4 \mathrm{~d}$, in contrast to the results with ATP. Correlations between oscillations of the two cyclic nucleotides in plasmodia starved for $1 \mathrm{~d}$ and $4 \mathrm{~d}$ are compared in Fig. 3(e, $f$ ). With starvation for $1 \mathrm{~d}$ there is no significant correlation (correlation coefficient 0.15 ). In a well-starved plasmodium the oscillations are in phase (correlation coefficient 0.78 ).

\section{Temperature independence of the period in the light-induced ATP oscillation}

Variation in the ATP concentration following illumination was measured at temperatures ranging from 10 to $28^{\circ} \mathrm{C}$. Even at $10^{\circ} \mathrm{C}$, as at higher temperatures, the ATP concentrations oscillated with a period of about $5 \mathrm{~h}$ and with a large amplitude (Fig. 4). The periods of lightinduced ATP oscillation at various temperatures (mean, SE and number of experiments, $n$, indicated) were $4.9 \pm 0.7 \mathrm{~h}(n=4)$ at $10^{\circ} \mathrm{C} ; 5.2 \pm 0.5 \mathrm{~h}(n=8)$ at $15^{\circ} \mathrm{C} ; 4.5 \pm 0.6 \mathrm{~h}(n=16)$ at $22^{\circ} \mathrm{C}$; and $4.3 \pm 0.5 \mathrm{~h}(n=11)$ at $28^{\circ} \mathrm{C}$. The period of the ATP oscillation hence was independent of temperature. Similar ATP oscillations were observed in an apogamic albino strain, LU 404 (Fig. 4b). ATP, cAMP and cGMP oscillations having similar period have been observed with growing plasmodia in relation to the cell cycle (Fink, 1975; Lovely \& Threlfall, 1976; Tyson 1982). Whether and how our oscillations described above are related to mitosis remains for further study.

We thank Dr M. J. Carlile of Imperial College, London, for revising the manuscript and for supplying the strain LU 404.

\section{REFERENCES}

Beutler, M. \& Baluda, M. (1964). Simplified determination of blood adenosine triphosphate using the firefly system. Blood 23, 688-698.

Chapman, A. \& CoOTE, J. G. (1982). Sporulation competence in Physarum polycephalum CL and the requirement for DNA replication and mitosis. Journal of General Microbiology 128, 1489-1501.

DANIEL, J. W. (1966). Light-induced synchronous sporulation of a myxomycete: the relation of initial metabolic changes to the establishment of a new cell 
state. In Cell Synchrony, pp. 117-152. Edited by I. L. Cameron \& G. M. Padilla. New York \& London: Academic Press.

Daniel, J. W. \& RusCh, H. P. (1962a). Methods for inducing sporulation of pure cultures of the myxomycete Physarum polycephalum. Journal of Bacteriology 83, 234-240.

Daniel, J. W. \& Rusch, H. P. (1962b). Niacin requirement for sporulation of Physarum polycephalum. Journal of Bacteriology 83, 1244-1250.

DEE, J. (1975). Slime moulds in biological research. Science Progress 62, 523-542.

FINK, K. (1975). Fluctuations in deoxyribo- and ribonucleoside triphosphate pools during the mitotic cycle of Physarum polycephalum. Biochimica et biophysica acta 414, 85-89.

Goodman, E. M. (1980). Physarum polycephalum: a review of a model system using a structure-function approach. International Review of Cytology 63, 1-58.

Gorman, J. A. \& Wilkins, A. S. (1980). Developmental phases in the life cycle of Physarum and related myxomycetes. In Growth and Differentiation in Physarum polycephalum, pp. 157-202. Edited by W. F. Dove \& H. P. Rusch. Princeton, USA : Princeton University Press.

GutTes, E., GutTes, S. \& Rusch, H. P. (1961). Morphological observation on growth and differentiation of Physarum polycephalum growth in pure culture. Developmental Biology 3, 588-614.
Honma, M., Satoh, T., Takezawa, T. \& UI, M. (1977). An ultrasensitive method for the simultaneous determination of cyclic AMP and cyclic GMP in small-volume samples from blood and tissue. Biochemical Medicine 18, 257-273.

Hosoda, E. (1980). Culture methods and sporulation of Physarum polycephalum. Mycologia 72, 500-504.

Knowles, D. J. \& Carlile, M. J. (1978). The chemotactic responses of plasmodia of the myxomycete Physarum polycephalum to sugars and related compounds. Journal of General Microbiology 108, $17-$ 25.

Lovely, J. R. \& Threlfall, R. J. (1976). Fluctuations in cyclic adenosine $3^{\prime}, 5^{\prime}$-monophosphate and cyclic guanosine $3^{\prime}, 5^{\prime}$-monophosphate during the mitotic cycle of Physarum polycephalum. Biochemical and Biophysical Research Communications 71, 789-795.

SAUER, H. W. (1982). Developmental Biology of Physarum, chapter 6. Cambridge: Cambridge University Press.

Sauer, H. W., Babcock, K. L. \& Rusch, H. P. (1969). Sporulation in Physarum polycephalum; a model system for studies on differentiation. Experimental Cell Research 57, 319-327.

Tyson, J. J. (1982). Periodic phenomena in Physarum. In Cell Biology of Physarum and Didymium. vol. 1, pp. 61-110. Edited by H. C. Aldrich \& J. W. Daniel. New York \& London: Academic Press. 